\title{
Shit happens - in Kliniken öfter am Wochenende
}

\section{Die stationäre Aufnahme ins Krankenhaus am Wochenende geht mit einer höheren Wahrscheinlichkeit für eine Hospitalinfektion, längerem Kranken- hausaufenthalt und höheren Kosten einher.}

— In den USA wurde 2001 der Begriff der "never events“ durch das nationale Qualitätsnormforum eingeführt. Dabei handelt es sich um schwerwiegende, kostspielige und vermeidbare medizinische Fehler, die eigentlich niemals passieren dürften. Nach Presseverlautbarungen in Dezember 2014 soll ein Rückgang dieser Fehler um 17\% in den Jahren 2010-2014 etwa 50.000 Leben gerettet und etwa 11 Milliarden Dollar eingespart haben.

$\mathrm{Zu}$ „never events“ gehören z. B. Luftembolien, vergessene Fremdkörper im Operationsgebiet, Übertragung nicht kompatibler Blutkonserven, Druckgeschwüre, katheterassoziierte Harnwegsinfekte, schlechte Blutzuckereinstellung, Stürze und Traumata, tiefe Venenthrombosen und Lungenembolien, iatrogener Pneumothorax sowie Wundinfektionen nach orthopädischen Ein- griffen, Implantation von elektronischen Geräten und bariatrischer Chirurgie.

Anhand einer nationalen Datenbank wurden die Daten von über 351 Mio. zwischen 2002 und 2010 in US-amerikanischen Krankenhäusern stationär behandelten Patienten analysiert. 19\% von ihnen wurden an einem Wochenende aufgenommen. Die Gesamtrate von im Krankenhaus erworbenen Problemen betrug 4,1\%. Dabei bestand ein signifikanter Unterschied zwischen den werktags aufgenommenen Patienten, von denen 3,7\% Komplikationen hatten, und den am Wochenende aufgenommenen mit einer Rate von 5,7\%.

Die Wahrscheinlichkeit, eine oder mehrere der genannten Komplikationen zu erleiden, war bei Aufnahme am Wochenende um $20 \%$ höher als während der Woche. Trat eine Komplikationen auf, bestand eine um $83 \%$ höhere Wahr- scheinlichkeit für höhere Kosten und eine um 38\% höhere Wahrscheinlichkeit für einen längeren Klinikaufenthalt.

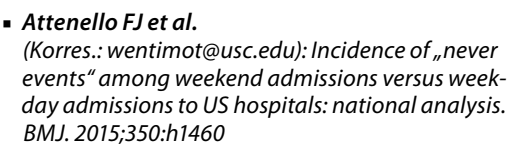

- Attenello FJ et al.

(Korres.: wentimot@usc.edu):Incidence of "never events" among weekend admissions versus weekday admissions to US hospitals: national analysis. BMJ. 2015;350:h1460

\section{Kommentar}

Jeder, der schon einmal im Krankenhaus gearbeitet hat, weiß, dass der Betrieb am Wochenende nicht so funktionieren kann wie während der Woche. Andernfalls wären alle Verwaltungsleiter schon auf die Idee gekommen, das Personal - das immerhin ca. 75\% des Gesamtbudgets „verschlingt” - auf ein Drittel zu reduzieren. Die Zunahme krankenhausspezifischer Komplikationen mag skandalös erscheinen, ist aber für Kenner der Verhältnisse ziemlich normal. Man bedenke auch, dass es am Wochenende mehr Notfälle und unvorhergesehene Ereignisse gibt, die naturgemäß mit schlechteren Behandlungsergebnissen und höheren Fehlerquoten einhergehen.

Prof. Dr. med. H. S. FüeßI .

\section{Seltene Komplikation von Kompressionsstrümpfen}

Ein 87-jähriger Mann mit Diabetes, Hypertonie, koronarer Herzkrankheit und Symptomen einer peripheren arteriellen Ver-

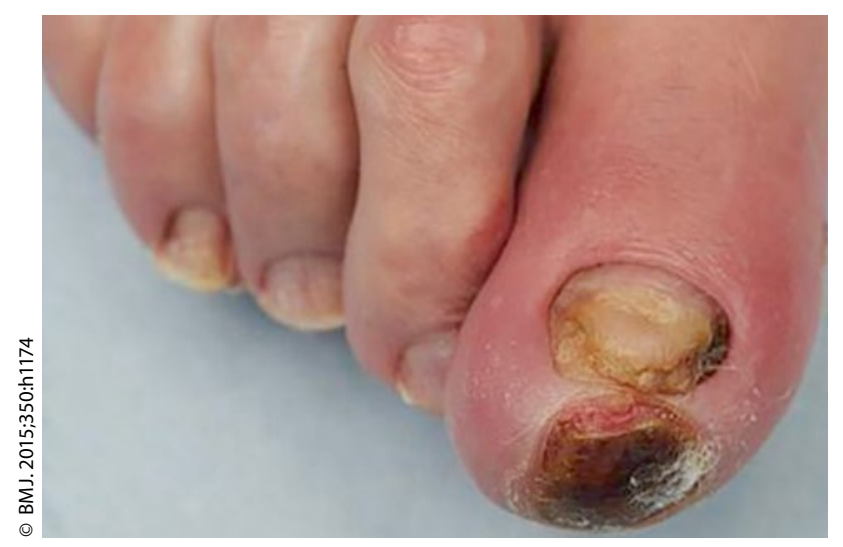

Nekrose an der Spitze der rechten Großzehe nach dem Tragen von Kompressionsstrümpfen im Krankenhaus. schlusskrankheit (pAVK) wurde wegen eines rechtsseitigen Kolonkarzinoms stationär zur Hemikolektomie aufgenommen. Gemäß den Vorgaben des Krankenhauses zur Prophylaxe thromboembolischer Ereignisse erhielt er niedermolekulares Heparin und Kompressionsstrümpfe. Postoperativ stellte man eine Nekrose an der Spitze der rechten Großzehe fest.

Patienten mit einem hohen Risiko für eine pAVK müssen vor Anpassung von Thrombosestrümpfen hinsichtlich ihrer Durchblutungsverhältnisse sorgfältig untersucht werden. Entsprechend der Leitlinie des National Institute for Health and Care Excellence aus dem Jahr 2010 sollten bei Vorliegen einer Durchblutungsstörung alternative Maßnahmen erwogen werden, z. B. eine pneumatische Kompression oder Fuß-Impuls-Technologie.

Prof. Dr. H. S. FüeßI .

\footnotetext{
- Karim A et al.

(Korres.: Dept. of Surgery, Heart of England Hospitals Trust, Birmingham B9 5SS): A rare complication of antiembolism stockings. BMJ. 2015;350:h1174
} 